\title{
PERFORMANCE EVALUATION OF MOBILE WIMAX IEEE 802.16E FOR HARD HANDOVER
}

\author{
Amira Youssef Fahoud and D.K. Lobiyal \\ School of Computer \& Systems Sciences, Jawaharlal Nehru University, New Delhi, India
}

\begin{abstract}
Seamless handover in wireless networks is to guarantee both service continuity and service quality. In WiMAX, providing scalability and quality of service for multimedia services during handover is a main challenge because of high latency and packet loss. In this paper, we created four scenarios using Qualnet 5.2 Network Simulator to analyze the hard handover functionality of WiMAX under different conditions. The scenarios such as Flag with 5 and $10 \mathrm{sec}$ UCD and DCD interval values, Random mobility scenario and DEM scenario using 6 WiMAX Cells have been considered. This study is performed over the real urban area of JNU where we have used JNU map for scenarios 1, 2 and 3 but for scenario 4, the JNU terrain data has been used. Further, each BS of 6 WiMAX cell is connected to four nodes. All nodes of each scenario are fixed except Node 1 . Node 1 is moving and performing the handover between the different BSs while sending and receiving real time traffics. Flag mobility model is used in Scenario 1, 2 and 4 to model the movement of the Node 1 while we use random mobility model in sceanrio3. 5 seconds time interval is used for Scenarios 1, 3, and 4 while 10 seconds time interval is used for scenario 2 to study the effect of management messages load on handover. Further, the statistical measures of handover performance of WiMAX in terms of number of handover performed, throughput, end-to-end delay, jitter, and packets dropped are observed and evaluated.
\end{abstract}

\section{KEYWORDS}

Wireless Broadband; WiMAX; Performance Evaluation; Qualnet; Handover

\section{INTRODUCTION}

Worldwide Interoperability for Microwave Access (WiMAX) brings broadband experience into wireless context [1]. It is an emerged industry based standard technology to provide a cost effective alternative solutions to the high cost wired broadband technologies available nowadays like DSL, T1/E1, and cable modems [2]. WiMAX is based on IEEE802.8 family of standards for providing wireless broadband connectivity over a metropolitan sized network and in two possible developments [3]. They are fixed WiMAX which is based on IEEE802.16d 2004 and Mobile WiMAX that is based on IEEE802.16e 2005 [4]. Broadband provides end users with certain benefits for traditional services and new multimedia services as well. Broadband systems must provide these benefits with a robust QoS in terms of throughput, jitter, End-to-End delay and packet error rate [2]. Mobility on the other hand is the most advantage provided by Mobile WiMAX to end users [5], but it brings main challenges like the need to address two important issues for supporting mobility; they are roaming and handover as well [6]. Roaming and Handoff are what we mobility management should take care of [7]. It should find means for supporting roaming and making seamless handover as well. As for a seamless handover, how to maintain ongoing sessions without it being interrupted while on the move even with a vehicular speed is a

DOI: $10.5121 / \mathrm{ijcnc} .2016 .8501$ 
serious challenge [8]. This serious challenge rises on how and when to make the transition process [9]. Algorithms which are responsible for taking the decision on when to make the handover needs to assure the balance between handoff rate and the dropping probability [10]. So, serving multimedia applications while on move and with a certain levels of QoS is more challenging because of the time variability and channel unpredictability and the situation became more critical when doing the handoff from one cell to another [10]. Handover in WiMAX is classified into three types; they are hard handover (HHO), Macro Diversity Handover (MDHO) and Fast Base Station Switching (FBSS) [10]. Hard handover is mandatory in WiMAX systems.

The other two types of handover are optional [10]. During hard handover, the MS communicates with only just one BS each time. Connection with the old BS is broken before the new connection is established with the new serving base station. In this paper we do a performance evaluation of the basic handover of WiMAX in multi-cell environment with high mobility for Real Time Traffic [11]. The contribution of this paper is to evaluate the performance of hard handover functionality of WiMAX under different conditions and in terms of number of handover performed, throughput, end-to-end delay, jitter, and packets dropped for real time applications.

The rest of this paper is organized as follow: section 2 defined the hard handover in WiMAX; section 3 outlines some of the related work done in this area of research. The detailed simulation setup has been described in section 4 , whereas section 5 explains the results analysis. Finally this study is summarized in section 6 .

\section{HARD HANDOVER}

The default handover in mobile WiMAX (IEEE 802.16e) is hard handover and the entire process of HHO is divided into two phases [10]. They are Network Topology Acquisition Phase (NTAP) and the Actual Handover phase (AHOP) [10, 12]. The procedures of handover consists of cell reselection through scanning, then taking the decision and initiation of handover, Finally network entry including the synchronization and ranging with the target base station [11]. The hard handover is depicted in the Figure.1. Selection is done by the MS and its serving base station with the help of network backbone. They will collect the information about network topology and Neighbour base stations. They identify the list of potential base stations around that could enter the process oh handover later. Out of this list one base station will be chosen to be target base station. The messages included in this phase are listed in the Table. $1[13,12]$

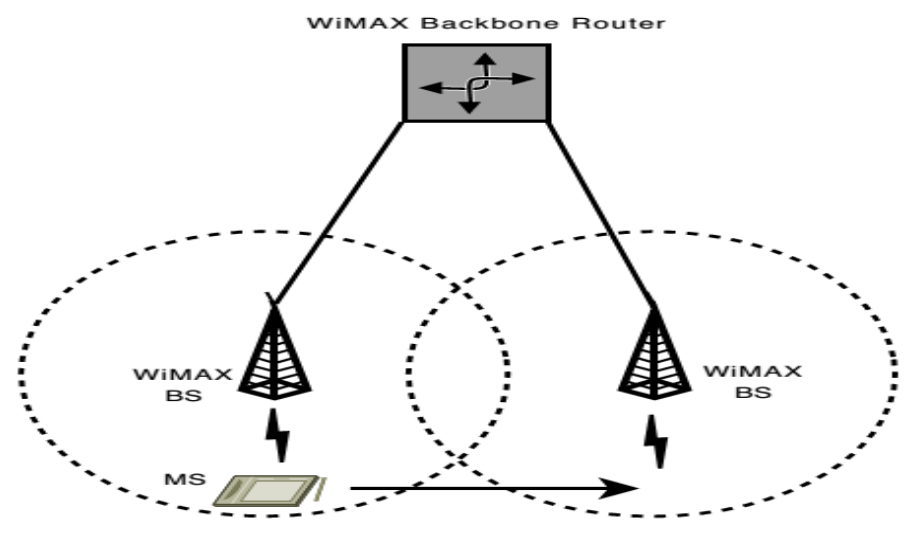

Figure 1: Hard Handover 
Table 1. Handover Messages Description.

\begin{tabular}{|l|l|}
\hline Message & Description \\
\hline MOB- NBR-ADV & $\begin{array}{l}\text { Mobile Neighbour Advertisement) } \\
\text { message, BS broadcasts information } \\
\text { about the state of the network base } \\
\text { stations periodically. }\end{array}$ \\
\hline MOB -SCN-REQ & Scanning request from MS \\
\hline MOB -SCN-RSP & Scanning response from BS \\
\hline MOB- SCN-REP & Scanning result Report \\
\hline RNG- REQ & Ranging Request from MS \\
\hline RNG -RSP & Ranging Response from BS \\
\hline MOB ASC-REP & Association Result Reports \\
\hline MOB MSHO-REQ & $\begin{array}{l}\text { From the MS to the Serving Base station } \\
\text { listing the target base stations. This } \\
\text { message will be sent in case the decision } \\
\text { of handover is made by the MS }\end{array}$ \\
\hline MOB BSHO-RSP & $\begin{array}{l}\text { BS reply back for the MOB MSHO-REQ } \\
\text { message. }\end{array}$ \\
\hline MOB BSHO-REQ & $\begin{array}{l}\text { From the BS to the Serving Base station } \\
\text { listing the target base stations. This } \\
\text { message will be sent in case the decision } \\
\text { of handover is made by the BS }\end{array}$ \\
\hline
\end{tabular}

\section{RELATED WORK}

Handover is an important issue to be tackled when providing Multimedia applications over wireless broadband networks. How to make the handover between WiMAX BSs was studied in [10] to ensure providing seamless handover for multimedia applications, S. K. Ray et al. presented the issues related to handover along with suggesting different solutions to face these issues and challenges. In [14] Pero et al. discussed the effect of handover on the performance of Mobile, WiMAX. They concluded that handover latency and dropping rate have a significant influence over the performance. In [15] Zina et al. in their paper studied handover issues and its effect on multimedia traffic, while presenting a MAC layer solution for optimizing handover for video applications in WiMAX. Their solution reduced the scanning time pre handover through the process of eliminating the number of scanned BSs according to different parameters like required bandwidth support. In [16] Po-wen Chi et al. proposed a fast and controlled handover scheme to decrease handover procedure and according to the results presented in the work the proposed scheme outperforms the default one. G. Khishigjargal et al. in their paper [17] defined the procedures of MBS handover. They evaluate the performance of WiMAX under conditions related to MBS handover for mobile IPTV in Qualnet simulator. Performance metrics are handover latency and data loss. 


\section{SIMULATION SETUP}

We created Four WiMAX scenarios using Qualnet 5.2 Network Simulator to test the basic handover functionality of IEEE 802.16e in multi_cell environment and, high mobility for real time applications. The created scenarios are composed of six subnets; each has 4 nodes connected with a BS. Nodes 4, 5, 10, 13, 17, and 21 in each scenario are the BSs of these six subnets, as it is shown in the Table 2

Table 2: Subnets

\begin{tabular}{|l|l|l|}
\hline Subnet & Nodes attached & Base Station \\
\hline 192.0 .6 .0 & (1 to 4$)$ & 4 \\
\hline 192.0 .7 .0 & (5 to 8$)$ & 5 \\
\hline 192.0 .8 .0 & (9 to 12$)$ & 10 \\
\hline 192.0 .9 .0 & (13 to 16$)$ & 13 \\
\hline 192.0 .10 .0 & (17 to 20$)$ & 17 \\
\hline 192.0 .11 .0 & (21 to 24$)$ & 21 \\
\hline
\end{tabular}

All the BSs connect to node 25 (ASN-Gateway) via wired point-to-point links. Each subnet operates with different radio frequency. Node 1 is under the study. This node transmits and receives real time traffic while it is moving across the six cells. We use two models for modelling Node 1 mobility. The models are flag mobility model and Random mobility model. in Flag Node 1 moves from left to right, then from right to left. It is originally close to BS node 4. So it registers with BS node 4 . When it moves to right, it will perform handover to BS node 5 , then handover to BS node 10, then handover to BS node 21, then handover to BS node 17, then handover to BS node 13, and finally handover to original BS node 4. While, in Random Mobility model it moves with minimum speed of $0 \mathrm{Mps}$ and maximum speed of $20 \mathrm{kmps}$ in random and straight lines. Time for pause is 10 seconds. These two mobility models are considered when we are dealing with Cartesian coordinate system but only flag mobility model is considered when we are dealing with latitude-Longitude coordinate system. Two values are considered for UCD (uplink channel descriptor) and DCD (downlink channel descriptor) management messages time interval, 5 and 10 with flag mobility model. These channel descriptors indicate modulation-code rate information of bursts for uplink and downlink respectively. 6 CBR flow are running to model the real time traffic in each Scenario as follow:

- CBR 1320001024 1S 10S 0S PRECEDENCE 3 (rtPS)

- CBR 1720001024 1S 10S OS PRECEDENCE 3 (rtPS)

- CBR 11420001024 1S 10S OS PRECEDENCE 3 (rtPS)

- CBR 18120001024 1S 10S OS PRECEDENCE 3 (rtPS)

- CBR 9120001024 1S 10S OS PRECEDENCE 3 (rtPS)

- CBR 22120001024 1S 10S OS PRECEDENCE 3 (rtPS)

So Node 1 is sending 3 CBR flows and Receive 3CBR Flows while it moves and performs handovers. Totally six handovers should be performed. The general Simulation parameters are listed in the Table.3: 
Table 3: General Simulation parameters

\begin{tabular}{|l|l|}
\hline Parameters & Values \\
\hline Length of simulation & 4 mintues \\
\hline Mobility Models & $\begin{array}{l}\text { Flag mobility (File based Mobility) used for } \\
\text { node } 1\end{array}$ \\
\hline Frequency Band (GHZ) & $2.4 \mathrm{GHz}$ \\
\hline Channel Bandwidth (MHZ) & 20 \\
\hline Frame Duration (ms) & 20 \\
\hline FFT Size & 2048 \\
\hline BS Transmitted Power (dbm) & 20 \\
\hline SS Transmitted Power (dbm) & 20 \\
\hline Simulation Time (s) & 240 seconds(4 M) \\
\hline Traffic & CBR \\
\hline Antenna Type & Omni-directional \\
\hline Radio Type & $802.16 \mathrm{e}$ \\
\hline Packet size & 1024 \\
\hline Base Station Antenna Height & 32 m \\
\hline MS Antenna Height & 1.5 m \\
\hline Neighbour BS Scanning RSS Trigger & -76 \\
\hline Handover RSS Trigger(dBm) & -78 \\
\hline Handover RSS Margin(dB) & 3 \\
\hline Cyclic Prefix Factor & 8 \\
\hline MAC frame Duration & 20 milli-second \\
\hline TDD Downlink Duration & 10 milli-secod \\
\hline DCD Broadcast Interval & 5 seconds \\
\hline UCD Broadcast Interval & 5 seconds \\
\hline Ranging Minimal Backoff Value & 3 seconds \\
\hline Ranging Maximal Backoff Value & 15 \\
\hline Service Flow Timeout Interval & 15 seconds \\
\hline Transmit/Receive Transition Gap(TTG) & 10 micro second \\
\hline Receive/Transmit Transition Gap( RTG) & 10 micro second \\
\hline SS Transition Gap(SSTG) & 4 micro second \\
\hline Maximum Allowed Uplink Load Level & 0.7 \\
\hline Maximum Allowed Downlink Load Level & 0.7 \\
\hline Bandwidth Request Minimal Backoff Value & 3 \\
\hline Bandwidth Request Maximal Backoff Value & 15 \\
\hline No of Packets sent for each application & $50,100,1000,10000$ \\
\hline UCD and DCD time intervals & 5,10 \\
\hline & \\
\hline
\end{tabular}

\section{NETWORK MODEL}

5.1. Scenario 1: is shown in Figure. 2 we have placed six BS over 2000 x 3000 area considered to be the area of JNU, Jawaharlal Nehru University. Node 1 is moving according to flag mobility model. The scenario specific parameters are listed in the Table.4. Results of scenario 1 is compared with results of scenarios 2, 3, 4 for studying the effect of load of management messages, Mobility model, terrain on the number of performed handover, respectively. 
International Journal of Computer Networks \& Communications (IJCNC) Vol.8, No.5, September 2016

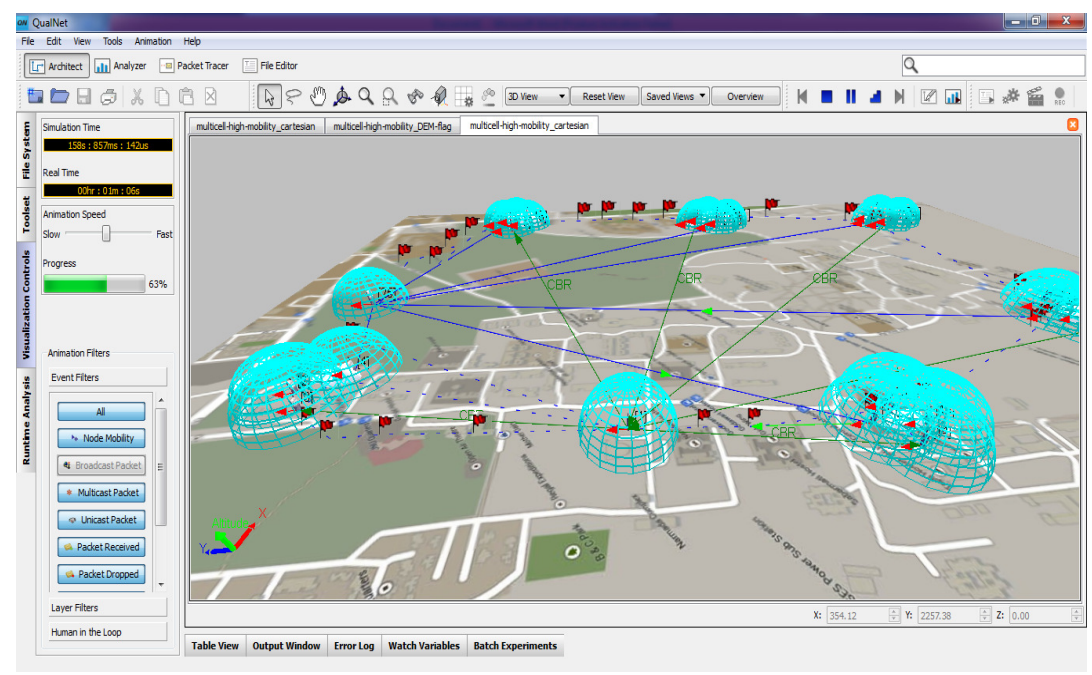

Figure 2: Scenario 1

Table 4: scenario 1 parameters

\begin{tabular}{|l|l|}
\hline Parameter & Values \\
\hline Coordinate system & CARTESIAN \\
\hline Coordinate Dimension & $2000^{*} 3000$ \\
\hline Terrain-Data-Type & Cartesian terrain data type \\
\hline Mobility Model & Flag Mobility Model \\
\hline UCD and DCD time interval & 5 \\
\hline
\end{tabular}

5.2. Scenario 2: is shown in Figure. 3 we have placed six BS over 2000 x 3000 area considered to be the area of JNU, Jawaharlal Nehru University. Node 1 is moving according to Flag mobility model. The scenario specific parameters are listed in the Table 5.

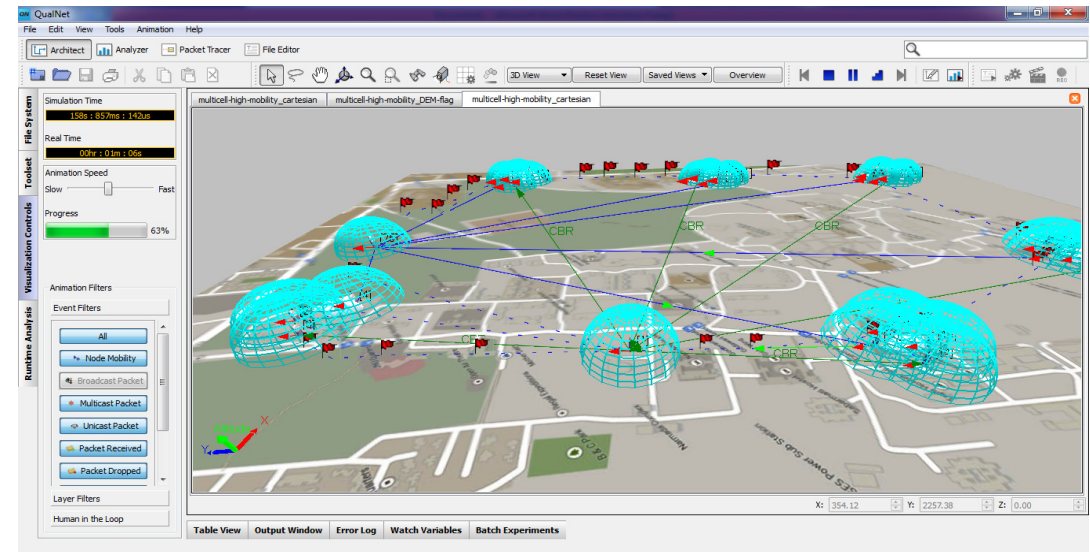

Figure 3: Scenario 2 
International Journal of Computer Networks \& Communications (IJCNC) Vol.8, No.5, September 2016

Table 5: scenario 2 Parameters

\begin{tabular}{|l|l|}
\hline Parameter & Values \\
\hline Coordinate system & CARTESIAN \\
\hline Coordinate Dimension & $2000 * 3000$ \\
\hline Terrain-Data-Type & Cartesian terrain data type \\
\hline Mobility Model & Flag Mobility Model \\
\hline UCD and DCD time interval & 10 \\
\hline
\end{tabular}

5.3. Scenario 3: is shown in Figure. 4 we have placed six BS over 2000 x 3000 area considered to be the area of JNU, Jawaharlal Nehru University, as the first Scenario but here Node 1 is moving according to the Random Mobility Models. The specific scenario parameters is listed in Table.6

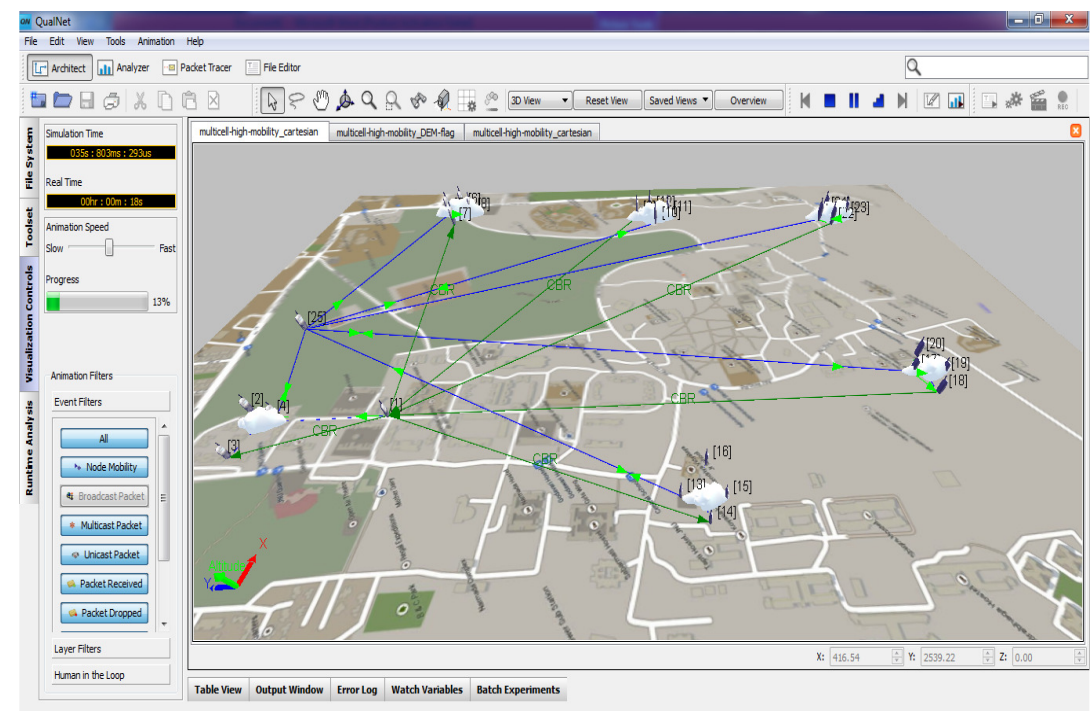

Figure 4: Scenario 3

Table 6: scenario 3 Parameters

\begin{tabular}{|l|l|}
\hline Parameter & Values \\
\hline Coordinate system & CARTESIAN \\
\hline Coordinate Dimension & $2000 * 3000$ \\
\hline Terrain-Data-Type & Cartesian terrain data type \\
\hline Mobility Model & Random Mobility Model \\
\hline UCD and DCD time interval & 5 \\
\hline
\end{tabular}


International Journal of Computer Networks \& Communications (IJCNC) Vol.8, No.5, September 2016

5.4. Scenario 4: is shown in Figure.5 we have placed six BS over the area of JNU. Using JNU DEM data for terrain. The specific scenario parameters are listed in the Table 7

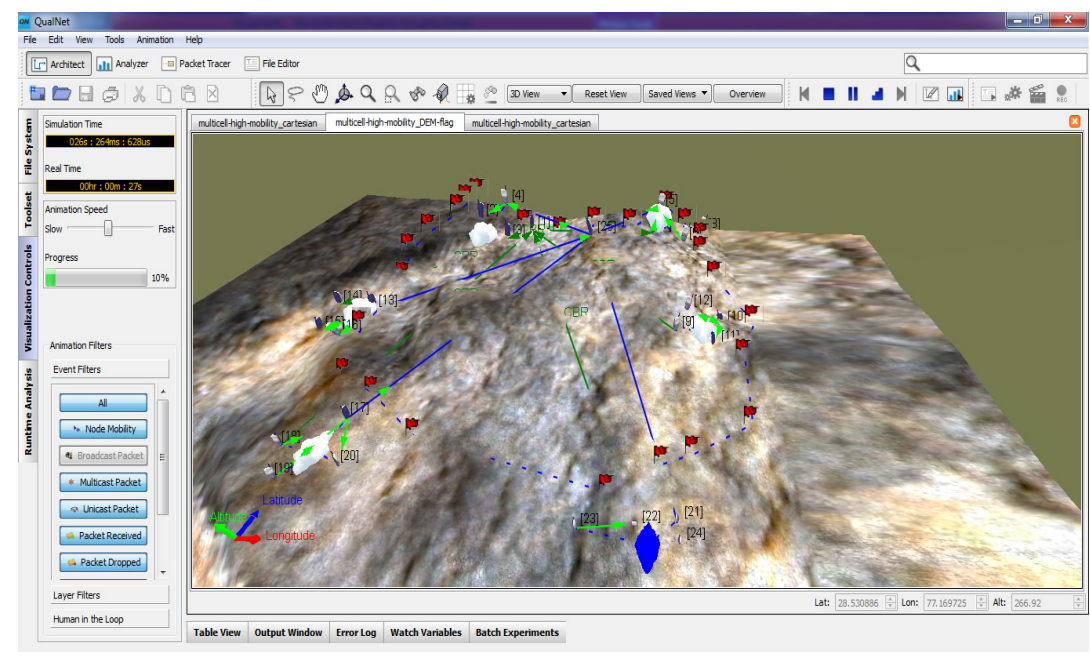

Figure 5: Scenario 4

Table 7: scenario 4 Parameters

\begin{tabular}{|l|l|}
\hline Parameter & Values \\
\hline Coordinate system & LATLONALT \\
\hline Terrain-south-west-corner & $(28.5251,77.1526)$ \\
\hline Terrain-North-East-corner & $(28.5536,77.1796)$ \\
\hline Terrain-Data-Format & $\begin{array}{l}\text { USGS DEM (Digital } \\
\text { Elevation Model data type } \\
\text { produced by USGS. It is 1 } \\
\text { degree file with elevation } \\
\text { points in a grid at } \\
\text { approximately 100 meters } \\
\text { spacing) }\end{array}$ \\
\hline Number of DEM Files & 1 \\
\hline DEM Terrain File & Jnu-DEM.dem \\
\hline Urban-Terrain-Format & None \\
\hline UCD and DCD time interval & 5 seconds \\
\hline Mobility Model & Flag Mobility Model \\
\hline
\end{tabular}




\section{RESULTS AND DISCUSSION}

We discuss simulation results, number of handover performed; throughput, end-to-end delay, and jitters as follow.

\subsection{Number of Handover}

Total number of successful handovers occurred during the simulation time $=$ NHO_success

Total number of failed handover during the simulation time $=$ NHO_fail

Total number of handover attempts during the simulation time $=$ Nattempt , where Nattempt $=$ NHO_success + NHO_fail

Table 8: Scenario 1- Cartesian-Flag Mobility Model-UCD and DCD-5

\begin{tabular}{|l|l|l|l|l|l|l|l|}
\hline Scenario 1: Cartesian-Flag Mobility Model-UCD and DCD-5 \\
\hline $\begin{array}{l}\text { No. of } \\
\text { Packets }\end{array}$ & 100 & 500 & 1000 & 1500 & 2000 & 2500 & $\begin{array}{l}30 \\
00\end{array}$ \\
\hline NHO_success & 3 & 5 & 5 & 5 & 5 & 5 & 5 \\
\hline NHO_fail & 1 & 1 & 1 & 1 & 1 & 1 & 1 \\
\hline Nattempt & 6 & 6 & 6 & 6 & 6 & 6 & 6 \\
\hline
\end{tabular}

Table 9: Scenario 2 - Cartesian-Flag Mobility Model-UCD and DCD-10

\begin{tabular}{|l|l|l|l|l|l|l|l|}
\hline Scenario 2 : Cartesian-Flag Mobility Model-UCD and DCD-10 \\
\hline $\begin{array}{l}\text { No. of } \\
\text { Packets }\end{array}$ & 100 & 500 & 1000 & 1500 & 2000 & 2500 & $\begin{array}{l}30 \\
00\end{array}$ \\
\hline NHO_success & 3 & 5 & 5 & 5 & 5 & 5 & 5 \\
\hline NHO_fail & 1 & 1 & 1 & 1 & 1 & 1 & 2 \\
\hline Nattempt & 6 & 6 & 6 & 6 & 6 & 6 & 6 \\
\hline
\end{tabular}

Table 10: Scenario 3- Cartesian-Random Mobility Model-UCD and DCD-5

\begin{tabular}{|l|l|l|l|l|l|l|l|}
\hline Scenario 3: Cartesian-Random Mobility Model-UCD and DCD-5 \\
\hline $\begin{array}{l}\text { No. of } \\
\text { Packets }\end{array}$ & 100 & 500 & 1000 & 1500 & 2000 & 2500 & 3000 \\
\hline NHO_success & 3 & 3 & 3 & 3 & 3 & 3 & 3 \\
\hline NHO_fail & 3 & 3 & 3 & 3 & 3 & 3 & 3 \\
\hline Nattempt & 6 & & 6 & 6 & 6 & 6 & 6 \\
\hline
\end{tabular}


Table 11: Scenario 4-DEM-Flag Mobility Model-UCD and DCD-5

\begin{tabular}{|l|l|l|l|l|l|l|l|}
\hline Scenario 4: DEM-Flag Mobility Model-UCD and DCD-5 \\
\hline $\begin{array}{l}\text { No. of } \\
\text { Packets }\end{array}$ & 100 & 500 & 1000 & 1500 & 2000 & 2500 & 3000 \\
\hline NHO_success & 2 & 2 & 3 & 3 & 3 & 3 & 3 \\
\hline NHO_fail & 4 & 4 & 4 & 3 & 3 & 3 & 3 \\
\hline Nattempt & 6 & 6 & 6 & 6 & 6 & 6 & 6 \\
\hline
\end{tabular}

In the first two scenarios (Table.8, Table.9) changing the interval time of receiving UCD and DCD is not affecting the number of handover performed. We start by sending 100 packets for each running application; we keep Node 1 busy in transmission and receiving till the end of simulation by changing the interval time between the generated packets. Since the interval for 100 is long, the node will have longer time of pausing before retransmitting, therefore the number of handover is less. By increasing the number of packets into 500 the number of handover performed is increased in the first two cases and remains constant while increasing the number of packets in the multiple of 500, i.e. 1000, 1500, 2000, 2500, and 3000. In case of Scenario 3 (Table.10), where the random mobility model is used, the number of handover performed is fixed for the varying number of sent Packets. It is due to the random movement of Node 1 and the position of node from the base station is affecting the possibility of executing handover. In scenario 4(Table.11), the number of handover is same for the first two cases where the number of packets sent was 100 and 500, and then it increased with increasing the number of packets sent and remained constant.

\subsection{Throughput}

Throughput refers to the rate of information arriving at or passing a particular point in the network. It is the total amount of data at that point divided by the time it takes to get the last packet. It is measured in bits per second [8] (bit/s or bps).

Throughput $=($ total bytes received $* 8) /($ time last packet received - time first packet received $)$

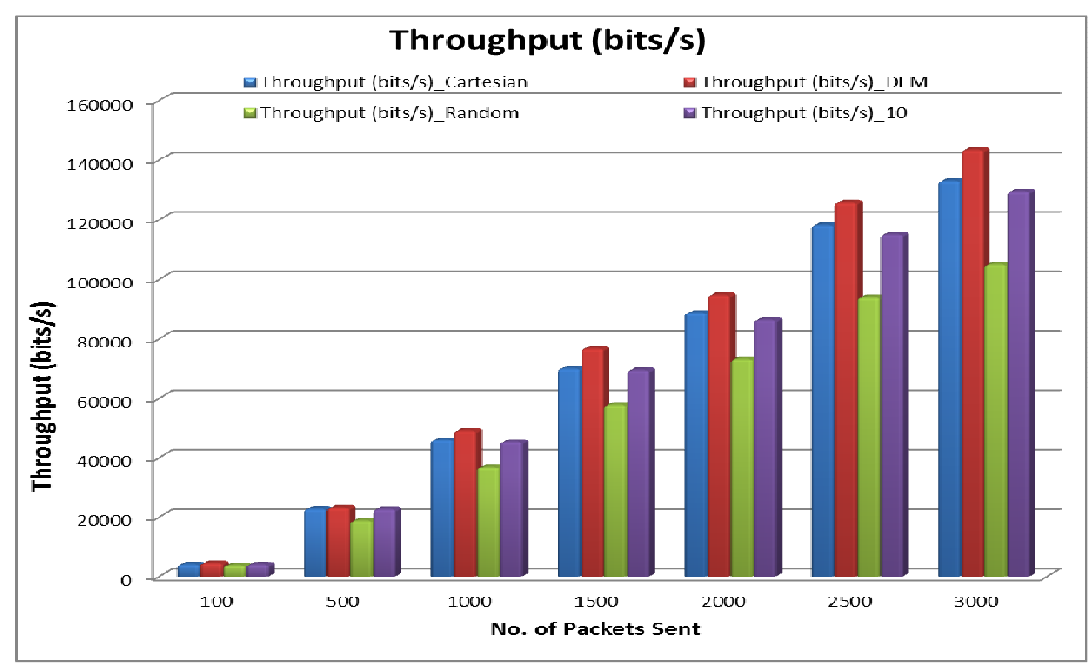

Figure 6: Throughput 
In case of Scenario 1 and 2 where the used value for UCD and DCD intervals are 5 and 10 seconds, the number of performed handover is same in varying number of packet sent shown in tables 7 and 8 . The only difference in both the scenarios was in throughput where throughput of scenario1 is higher than throughput of scenario 2, due to the number of packets dropped in 10sec is more compared to $5 \mathrm{sec}$ depicted in Figure 6.

The throughput for scenario-3 where random mobility model is used to model the movement of Node1, is less than throughput in Scenario 1 with flag mobility model, shown in figure 6, because the node 1 in Scenario-3 is getting away from the base stations and failing to make handover, therefore the larger number of packets are dropped.

In case of Scenario-4 where DEM data is considered, its throughput is higher than the throughput in Scenario 1 where the elevation of node is not taking into consideration.

\subsection{Average Unicast Jitter}

Jitter can seriously affect the quality of services. "It is the variation in delay of different data packets that reach the destination. For varying number of packets the jitter is more when the number of packet sent is less. From the Figure.7 the value of jitter in all scenarios is very high when 100 packets are sent, then it goes down when 500 packets are sent, and it becomes almost constant with increasing the number of packets, i.e. 1000, 1500, 2000, 2500, and 3000. In case of sending 100 and 500 packets the interval between the packets sent is large which causes the interval between the variations in delay of the received packets to be larger than the other cases of number of packets sent where the interval between packets sent is smaller.

In scenario-1 the jitter in case of 100 packets is less than jitter in Scenarios 2, 3 and 4 then it goes higher than scenario 2 and 3 in case of 500 packets sent, but for 1000 packets, jitter becomes less than both scenarios 2 , and 3 . Then it remains constant for varying number of packets.

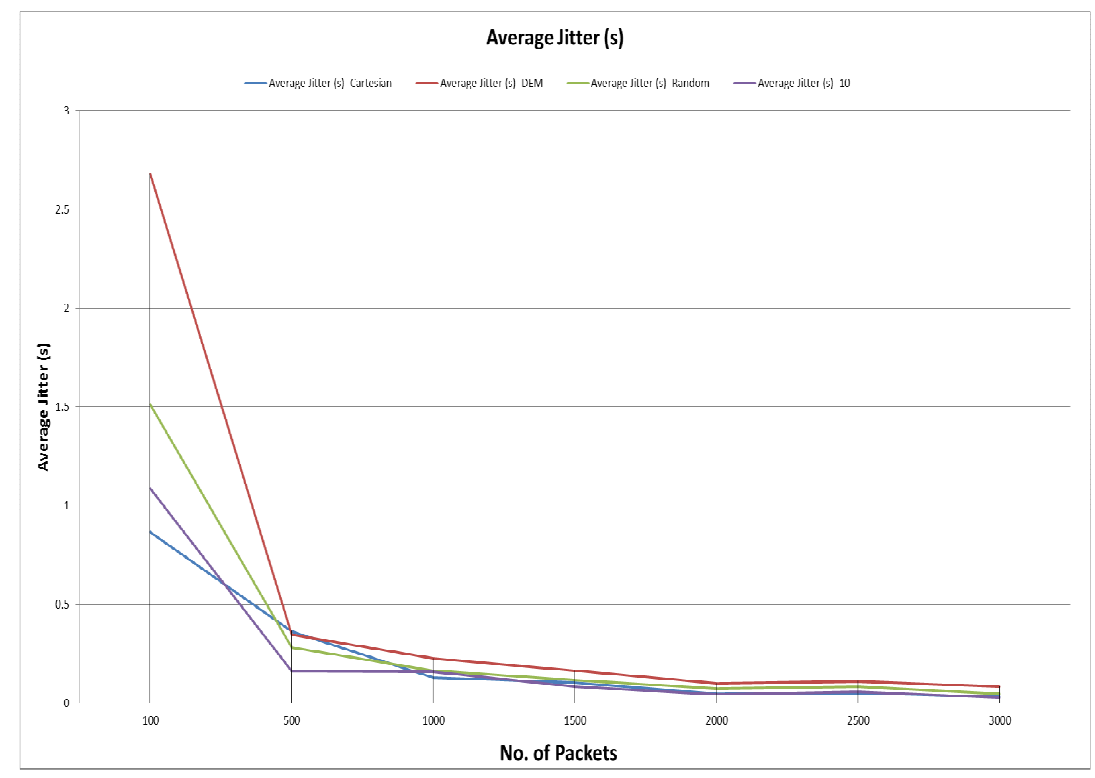

Figure 7: Jitter 
Jitter in scenario 2 is higher than jitter in scenario 1 only for 100 packets, and then it goes down for the varying number of packets.

In scenario-3 the number of handover performed is not changing at all along with changing the number of packets sent, but the jitter is higher in case of 100 and 500 packets sent. Whereas, scenario-4, has always higher jitter compare to the other scenarios, because of the variation in the terrain and then the variation in the delay of received data. Similar to other scenarios, scenario 4 also remains constant for the varying number of packets after 500 packets sent.

\subsection{End-To-End Delay}

End-to-end delay indicates how long or how much time units it takes for a packet to travel from the source to the destination. "It is the average data delay an application experiences while transmitting data given by [8].

\section{The average end-to-end delay is calculated as follows:}

Delay $=($ Total of packet delays for all packets) $/$ (Total packets received) Packet delay $=($ time when packet is received at the server - time when the packet is transmitted at the client)

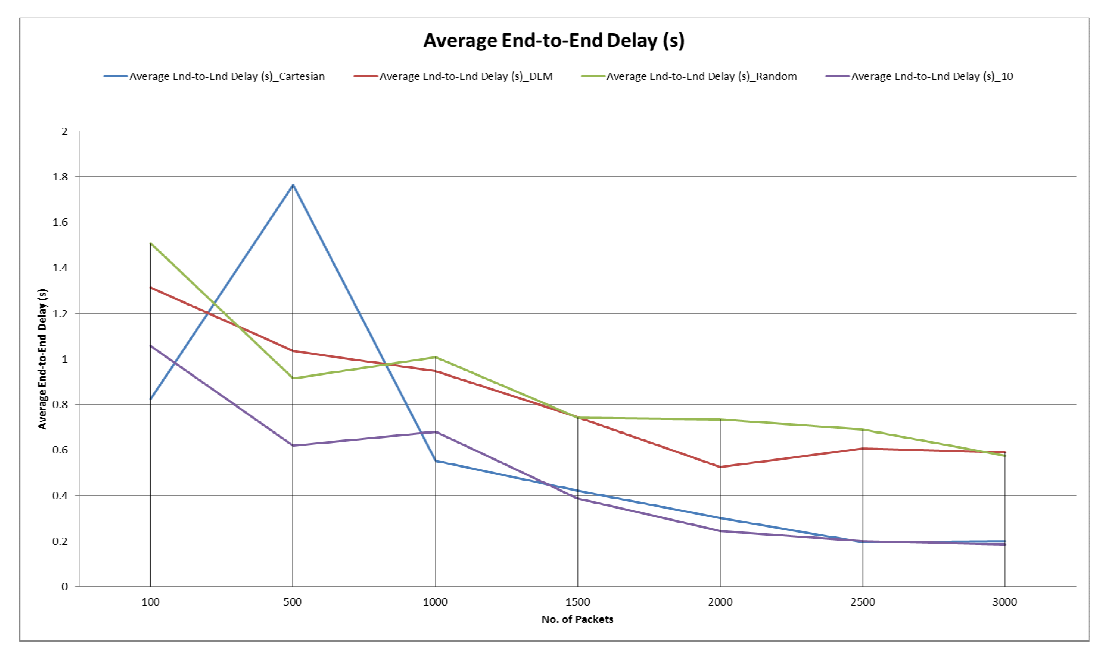

Figure 8: End-to-End Delay

Initially end-to-end delay (Figure.8) of scenario 1 is low compare to other scenarios in 100 number of packets sent, but it goes very high when the number of packets increase to 500. Later it fluctuates for the remaining varying number of packets, therefore, there is no pattern performed by scenario 1 . Similarly in the case of scenario 2,3 and 4 , end-to-end delay fluctuate with the varying number of packets, thus it is difficult to conclude the significance of mobility mode, UCD and DCD interval values and terrain on the network performance in terms of delay. In all scenarios the values of delay is higher to support rtPS data. 


\subsection{Packet Dropped}

Packet loss is another important performance metric for handover, in scenario-1, the packets dropped is less than the other scenarios in all the cases, whereas, scenario 3 has the highest packets dropped (Figure.9).

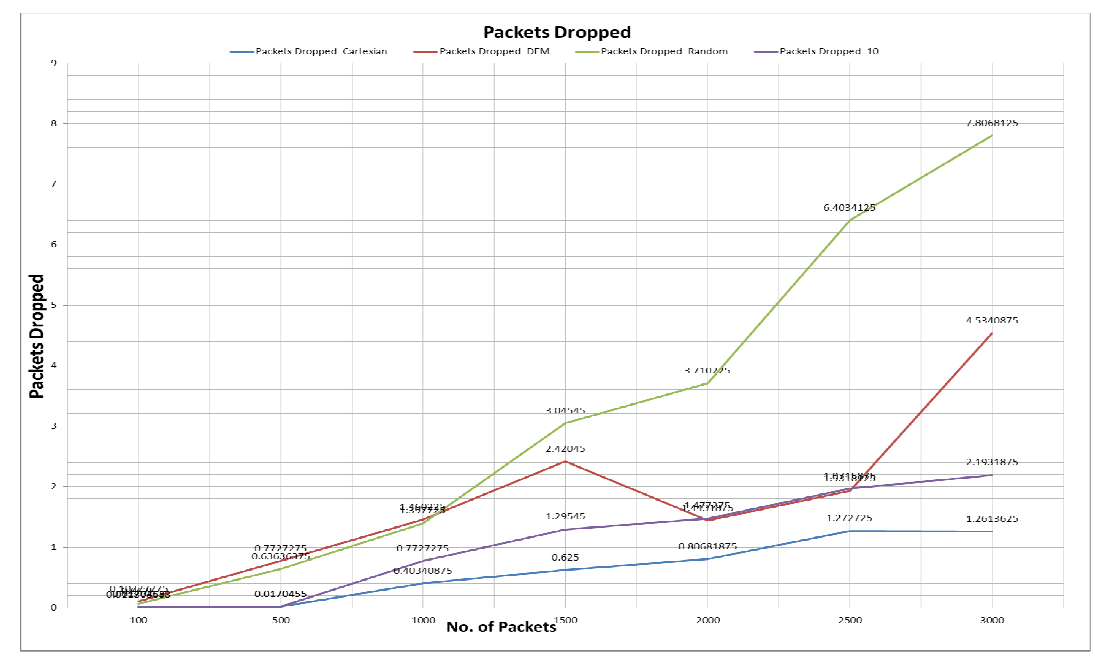

Figure 9: Packets Dropped

Scearnio-3 is the only scenario, whose number of packets dropped increased with the varying number of packets till 1500, after that it fluctuate. The number of dropped packets in scenarios 1 , 2 and 4 is increasing with the varying number of packets.

\section{CONCLUSIONS}

In this study, the real urban area that is our university, JNU is considered for the WiMAX handover performance. Four scenarios such as Flag with 5 and $10 \mathrm{sec}$ UCD and DCD interval values, Random mobility scenario and DEM scenario have been created using Qualnet Simulator. Further, the statistical measures of handover performance of WiMAX in terms of number of handover performed, throughput, end-to-end delay, jitter, and packets dropped are observed and evaluated. The obtained results indicate that the performance of handover in terms of number of handover performed and packets dropped is affected by the load of management messages, mobility model of the moving nodes and the variation of terrain data. Depending on this analysis we see that handover is affected by the mobility pattern and terrain variation more the load of management messages. The delay, jitter and packets dropped are high to support real-time application.

\section{REFERENCES}

[1] Rao, GSV Radha K., and G. Radhamani. WiMAX: a wireless technology revolution. CRC Press, 2007.

[2] Andrews, Jeffrey G., Arunabha Ghosh, and Rias Muhamed. Fundamentals of WiMAX: understanding broadband wireless networking. Pearson Education, 2007. 
[3] Etemad, Kamran. "Overview of mobile WiMAX technology and evolution." IEEE Communications Magazine 46.10 (2008): 31-40.

[4] Eklund, Carl, et al. "IEEE standard 802.16: a technical overview of the WirelessMANTM air interface for broadband wireless access." IEEE communications magazine 40.6 (2002): 98-107.

[5] Ahmadi, Sassan. "An overview of next-generation mobile WiMAX technology." IEEE Communications.

[6] Li, Bo, et al. "A survey on mobile WiMAX [wireless broadband access]."IEEE Communications magazine 45.12 (2007): 70-75.

[7] WiMAX-Part, Mobile. "I: A technical overview and performance evaluation. "WiMAX Forum (2006): 9-24

[8] Jain, Raj. "WiMAX system evaluation methodology v2. 1." WiMAX Forum. 2008.

[9] Jiao, Wenhua, Pin Jiang, and Yuanyuan Ma. "Fast handover scheme for real-time applications in mobile WiMAX." 2007 IEEE International Conference on Communications. IEEE, 2007: 6038-6042

[10] Ray, Sayan Kumar, Krzysztof Pawlikowski, and Harsha Sirisena. "Handover in mobile WiMAX networks: the state of art and research issues." IEEE Communications Surveys \& Tutorials 12.3 (2010): 376-399.

[11] Ben-Mubarak, Mohammed A., et al. "Movement direction-based handover scanning for mobile WiMAX." The 17th Asia Pacific Conference on Communications. IEEE, 2011.: 737-742

[12] Chen, Jenhui, Chih-Chieh Wang, and Jiann-Der Lee. "Pre-coordination mechanism for fast handover in WiMAX networks." The 2nd International Conference on Wireless Broadband and Ultra Wideband Communications (AusWireless 2007). IEEE, 2007.:15

[13] Chen, Ling, et al. "A cross-layer fast handover scheme for mobile WiMAX."2007 IEEE 66th Vehicular Technology Conference. IEEE, 2007.: 1578-1582

[14] Latkoski, Pero, and Borislav Popovski. "Evaluation of Mobile WiMAX handover procedure." Melecon 2010-2010 15th IEEE Mediterranean Electrotechnical Conference. IEEE( 2010): 476-481

[15] Jerjees, Zina, and Hamed Al-Raweshidy. "Handover Optimization for Video Applications in WiMAX." 2009 Third International Conference on Next Generation Mobile Applications, Services and Technologies. IEEE(2009): 189-196

[16] Lin, Yu-Hsiang, et al. "Fast Uncontrolled Handover scheme for WiMAX: infrastructure networks." International Journal of Autonomous and Adaptive Communications Systems 4.3 (2011): 237-251.

[17] Gonchigsumlaa, Khishigjargal, Young-il Kim, and Enkhzul Doopalam. "Performance analysis of MBS handover for mobile WiMAX." Advanced Communication Technology (ICACT), 2012 14th International Conference on. IEEE, 2012.29-32

\section{AUTHORS}

Amira is pursuing Ph.D in Computer Science and Technology from School of Computer and Systems Sciences, Jawaharlal Nehru University (JNU), New Delhi, India. She pursued her M.Tech (Computer Science and Technology) from JNU, in 2011, Information Engineering from Tishreen University, Lattakia, Syria in 2007 .Her area of interest includes Computer Networks, Wireless Networks, and broadband Networks.

Daya K. Lobiyal received his Ph.D. and M.Tech. (Computer science) from School of Computer and Systems Sciences, Jawaharlal Nehru University, New Delhi, India in 1996 and 1991, respectively. B.Tech. (Computer Science and Engineering) was pursued from Lucknow University, India, 1988. Presently, he is an Associate Professor in the School of Computer and Systems Sciences, Jawaharlal Nehru University, New Delhi, India. His research interest includes Mobile Ad hoc Networks, Vehicular Ad Hoc Networks, Wireless Sensor Network, Video on Demand and Natural Language

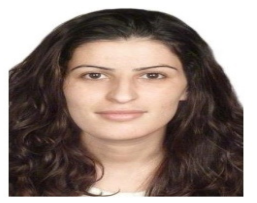
Processing

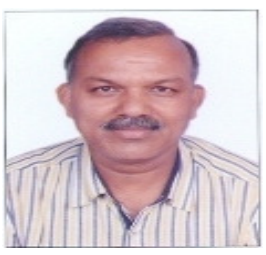

